\title{
Casimir Torque for a Perfectly Conducting Wedge: A Canonical Quantum Field Theoretical Approach
}

\author{
M. Abdollahi · H. Razmi
}

Received: 26 June 2007 / Accepted: 4 September 2007 / Published online: 11 October 2007

(C) Springer Science+Business Media, LLC 2007

\section{Erratum to: Int J Theor Phys \\ DOI 10.1007/s10773-005-1516-0}

Considering the paper by H. Razmi and S.M. Modarresi [1], the final result for Casimir torque is (mathematically) corrected. An explanation on the difference between the calculation for canonical scalar fields and the electromagnetic fields for the special geometry of wedge is presented.

Referring to the original article [1], because of some mathematical typos from relation (9) on, the relations (14) and (15) should be changed to:

$$
\begin{gathered}
\left\langle\left. T^{\varphi \varphi}\right|_{\varphi=0, \beta}\right\rangle=\lim _{\xi \rightarrow 1}\left(\sum_{m=1}^{\infty} \frac{\hbar c}{2 \beta \pi \rho^{4}}\left(\frac{m^{2} \pi^{2}}{\beta^{2}}\right)\right) \frac{\xi^{\left(\frac{m \pi}{\beta}\right)}}{\left(1-\xi^{2}\right)} \\
\left\langle\left. T^{\varphi \varphi}\right|_{\text {ren. }}\right\rangle=\lim _{\xi \rightarrow 1}\left[\left(\sum_{m=1}^{\infty} \frac{\hbar c}{2 \beta \pi \rho^{4}}\left(\frac{m^{2} \pi^{2}}{\beta^{2}}\right) \frac{\xi^{\left(\frac{m \pi}{\beta}\right)}}{\left(1-\xi^{2}\right)}\right)-\left(\sum_{m=1}^{\infty} \frac{\hbar c m^{2}}{2 \pi^{2} \rho^{4}} \frac{\xi^{m}}{\left(1-\xi^{2}\right)}\right)\right] .
\end{gathered}
$$

Thus, with a careful Mathematical calculation/computation (using Mathematica 5 program), the relations (16) and (17) in [1] are changed as:

The online version of the original article can be found under doi:10.1007/s10773-005-1516-0.

\footnotetext{
M. Abdollahi $(\bowtie) \cdot$ H. Razmi

Department of Physics, The University of Qom, Qom, Iran

e-mail: mah.abdollahi@gmail.com

H. Razmi

e-mail: razmi@qom.ac.ir

H. Razmi

e-mail: razmiha@hotmail.com
} 


$$
\begin{gathered}
\left\langle\left. T^{\varphi \varphi}\right|_{\text {ren. }}\right\rangle=\frac{-\hbar c}{480 \pi^{2} \rho^{4}}\left(\frac{\pi^{4}}{\beta^{4}}-1\right), \\
N=\frac{-1}{\rho} \frac{\partial}{\partial \beta}\left\langle\left. T^{\varphi \varphi}\right|_{\text {ren. }}\right\rangle=\frac{-\pi^{2} \hbar c}{120 \rho^{5} \beta^{5}},
\end{gathered}
$$

where, contrary to what was done in [1], here we have not multiplied by a factor of 2 to find the corresponding result for the electromagnetic fields. The reason is that for the wedge geometry we cannot obtain the force/torque due to the electromagnetic fields fluctuations simply by multiplying the result for the scalar fields by a factor of 2 (for a more detailed explanation about this specific property of the wedge geometry see [2]). Of course, in the asymptotic limit of the two parallel plates (the subject of the final section of [1]), the famous Casimir result for the electromagnetic force can readily be obtained by multiplying the above result by a factor of 2 .

\section{References}

1. Razmi, H., Modarresi, S.M.: Int. J. Theor. Phys. 44, 229 (2005)

2. Deutsch, D., Candelas, P.: Phys. Rev. D 20(12), 3063 (1979) 